\title{
Radionovela: lágrimas, susurros y una felicidad siempre pospuesta
}

\author{
Radio drama: tears, whispers and happiness always postponed
}

\author{
Oneibys Torres Figueroa \\ Universidad Central "Marta Abreu" de las Villas \\ oneibys@telecubanacan.icrt.cu \\ Elizabeth González Ocaña \\ Universidad Central "Marta Abreu" de las Villas. \\ egocana@uclv.edu.cu
}

Recibido: 11 de noviembre 2011

Aceptado: 29 de diciembre 2011

\section{Resumen}

El presente artículo dedicará sus páginas a las que tuvieron lugar en las ondas radiales; al espacio que todavía hoy hace llorar y reír a muchos en Cuba y a no tantos en el resto de América Latina: la radionovela.

Se pretende realizar un recuento de sus antecedentes, el género al que pertenece; qué condiciones económicas le permitieron escalar y posicionarse, incluso, como un renglón exportable más ¿Qué factor psicológico la mueve y la clasifica como un elemento de la industria cultural, y finalmente, qué la hace sobrevivir con salud en nuestro país, estar en la preferencia dentro de los dramatizados y convertir aún después de tantos años las lágrimas en placer.

En aras de lograrlo resulta obligatoria la consulta de fuentes primarias como sondeos de opinión realizados por la emisora provincial villaclareña CMHW; y secundarias como libros dedicados a la radiodifusión en sentido general, otros específicamente a la dramaturgia y la radionovela, además de algunos dedicados a testimonios de sus principales protagonistas. Otras técnicas de investigación utilizadas fueron la entrevista a una personalidad del género dramatizado, y la realización de sesiones de trabajo con grupos de personas mayores de treinta años, estratificados por su ocupación laboral.

\begin{abstract}
The present article will dedicate its pages to that which took place in the radial waves; to the space that today still makes many in Cuba, and not so many in the rest of Latin America, cry and laugh: the radio-opera.

A recount of their antecedents will be done, the genre to which it belongs; what economic conditions allowed it to climb and to be positioned, even, as an exportable line. What
\end{abstract}


psychological factor moves and classifies it as an element of the industry of culture, and finally, what makes it survive in a healthy way in our country, to be in the preference inside the dramatized programs and to still turn the tears into pleasure after so many years.

For the sake of achieving it, consultation of both primary sources, as opinion polls carried out by the provincial radio station in Villa Clara, CMHW is mandatory and secondary as books dedicated specifically to broadcasts in general, others specifically the drama and the radiooperas, besides some dedicated to testimonies of its main characters. Other used investigation techniques were an interview to a personality of the gender of drama, and the realization of work sessions with groups of people older than thirty years of age, stratified by their labor occupation.

Palabras clave: Radionovela, Emisora provincial, Radio.

Key words: Radio drama, Provincial station, Radio.

Sumario: 1. La radionovela, un producto que se resiste a abandonar los aparatos receptores. 2. Conclusiones. 3 Notas. 4. Bibliografía.

Summary: 1. The radio, a product that refuses to leave the receivers. 2. Conclusions. 3. Notes. 4. Bibliography.

\section{La radionovela, un producto que se resiste a abandonar los aparatos receptores}

El siglo XX prometía ser diferente en cuanto a la relación del hombre y la técnica. A partir de los inicios de la centuria la compañía del ser humano variaría, no sólo estaría rodeado de otros hombres, sino además del cine, la radio, y un poco más tarde, la televisión. Los pasajes familiares, amorosos, tristes, alegres, dejan de ser individuales, íntimos, personales, para formar parte de todos. Historias de diferente índole colmarían el habla popular, y en algunos casos pasarían a convertirse en leyendas que llegan a nuestros días.

La radionovela no siempre fue como la conocemos hoy día. Sus inicios están marcados, como diría Reinaldo González, por "su progenitor, el radioteatro, y su ancestro ilustre el folletín francés por entregas". Por algunos años la traslación de diálogos y acotaciones teatrales a la radio constituye, junto a los noticieros, la programación hablada de las emisoras latinoamericanas.

El teatro radiado se convierte en éxito rotundo. Las plantas que deseaban tener arraigo popular debían contar con su propio radioteatro (1). La radio era en sí misma más cómoda y accesible que los libros, el periódico y el cine, debido a que la mayor parte de los oyentes eran analfabetos y pobres. Este fue uno de los elementos que ayudó a impulsar a los 
radioteatros y posteriormente a las novelas radiales. El hecho de haber sido su progenitor ha determinado que en muchos países de nuestra región se conozcan como tal a los espacios que transmiten radionovelas. Del folletín adquiere, según Reinaldo González: lo exagerado, lo picante, lo crudo, lo exótico, el estereotipar acciones y caracteres, la costumbre de interrumpir cada capítulo en un efecto que despierte avidez por el próximo. Al decir de este autor, el conjunto resulta inorgánico, los personajes, como los de los malos folletinistas franceses decimonónicos, mudan de opinión y muestran una inconsistencia total en sus caracteres; también aquí son reemplazados por figuras secundarias que «crecen» hasta ganar el favor de la audiencia.

Las características heredadas de estos dos estilos circunscriben a la radionovela en el género dramático pues este: "tiene relación con los valores, con la ética. ¿Quién tuvo la culpa, quien tiene la razón? ¿Quién es el malo y quién es el bueno? Esas preguntas son fundamentales en todo argumento dramático. El drama es un género de ficción: muchas veces se inspira en hechos reales, pero trabaja con lo que podría pasar. Eso sí, aunque estamos en el terreno de la imaginación y la fantasía, la acción que representa debe ser verosímil, pudo haber pasado. Es creíble." (2).

Algo más que el estilo le llegó a la novela radial del folletín y es la concepción de la obra como mercancía, dejando a un lado los requerimientos literarios que exige, para dar un mayor valor a la tarifa, los precios, las fechas fijas de entrega según modelos preelaborados, y todo marcado por el interés comercial en detrimento de lo cultural.

Por supuesto que las agencias de publicidad no permanecieron de brazos cruzados ante esta situación. De inmediato desplegaron campañas que llegaron a sectores ante excluidos de su alcance y que ofrecen un motivo más para convertir "cada tema en un melodrama, independientemente de su sentido original". (3). El fenómeno incapacita a este formato dramatizado para transmitir variantes estilísticas, pero no para complacer todo tipo de gustos y llegar desde bodegueros hasta médicos, catedráticos, sacerdotes, psiquiatras y políticos.

El radioteatro estuvo destinado a morir, entre otras causas, por escudarse en empresas minoristas y desarrollarse en un período de crisis económica, a saber, finales de los años veinte y principios de los treinta. Ese no sería el destino de la radiodifusión que llegaría a ser: "un negocio de peso y de pesos, más bien de millones" (4) y dentro de ella la radionovela sería uno de los exponentes más importantes. "López del Rincón, después de múltiples ensayos, populariza Por la ciudad rueda un grito. Le siguen otros autores con obras como Aves sin nido, El collar de lágrimas, La novela del aire y El derecho de nacer, que marca una etapa de la novela radial. El estilo declamatorio va desapareciendo y da paso a una interpretación más natural. Como antecedente de la novela campesina está Pepe Cortés que 
inicia el autor Aramís del Real y termina el escritor Juan Domínguez Arbelo por la CMHI de Santa Clara." (5). Esta cita demuestra el rápido avance del género, en cuanto a la producción y la variación en el estilo. Inicialmente fue difícil lanzarla, pero pronto su paso sería dinámico en todo momento.

Ya en los años treinta del siglo XX, la radionovela era un producto comercial bien definido y a la vez conocido en América Latina. No tenía nada que envidiarle a la soap - opera (su pariente norteamericana), pues las empresas jaboneras le brindarían todo el apoyo que fuera necesario para dejar su nombre grabado en la historia de la radiodifusión en este país. La novela radial cubana se encontraba lista para marcar pautas de todo tipo en el serial dramatizado del continente, y por qué no, del resto del mundo. "(...) La producción de radionovelas cubanas se diversifica y crece hasta cubrir casi todas las horas de transmisiones radiales. Un país de reconocida tradición musical reduce su programación radiofónica a melodramas y «Guantanameras» en dejación de otros ritmos populares. La prensa de esos años recoge la protesta de los músicos que demandan mayor tiempo en el horario. (...) El melodrama radiofónico aumenta en Cuba en una proporción desconocida hasta entonces por otro país del continente. Por acumulación, en poco tiempo la isla caribeña incluye entre sus renglones exportables (...) un surtido de argumentos radiales capaces de irritar los más resistentes lagrimales." (6). Esta exportación adquiere tal auge que en pocos años la "realidad cubana", materializada en guiones radiales, está en los radiorreceptores de la mayoría de los países latinoamericanos, al punto de sofocar la producción nacional de algunos; y junto con estos, muchas veces, a sus escritores y directores.

Pero no sólo en el resto de América Latina se evidenciaba la influencia del paso de la radionovela, Cuba también daba muestras de sus efectos. Estos se hicieron evidentes en una contienda de la que eran protagonistas por igual la radionovela y la industria jabonera. Con ella se marcarían pautas para el posterior desarrollo de este género en el medio, esta fue nada más y nada menos que "una de las campañas económicas más violentas que se conocen en Cuba por esos años" (7) popularizada como "La Guerra del Aire" y en la cual CMQ le asesta un duro golpe a la RHC al lograr la captación del -por entonces ya reconocido- Félix B. Caignet, quien en los años venideros daría mucho de que hablar.

Se hace necesario hacer una pausa en el análisis de la radionovela para dedicarle unas oraciones al creador del melodrama más famoso de la historia de las novelas radiales, y uno de los mayores negociantes del medio. De él diría Enrique Núñez Rodríguez: "el padre indiscutible de la novela radial. Autor de Frutas en el Caney. De estampas costumbristas. De Te Odio, canción antológica. Su obra cumbre: El derecho de nacer. El más criticado de los 
autores radiales cubanos. Su frase inolvidable: "La envidia es admirar con rabia". Fue muy admirado."

Hablar de la radionovela, incluso de la radiodifusión en Cuba sin mencionar a Félix B. Caignet, es saltarse una parte muy importante de su historia, pues "fue uno de los primeros en concebir guiones totalmente radiofónicos y en introducir al narrador de radionovelas. Su trabajo marca pautas que deben seguir quienes aspiren al éxito después de él." (8). Desde el principio su obra fue muy bien aceptada por el público. En 1937 la radio cubana dota al hemisferio de un acontecimiento inicial de gran significación en la vida del serial detectivesco escrito especialmente para las ondas hertzianas: La Serpiente Roja, que introduce al inefable detective chino Chan Li Po con -más de ocho años de éxito delirante según Reinaldo González-, que empujan a su autor hacia el camino de la fama.

El Derecho de Nacer fue lanzada al aire el primero de abril de 1948, y aunque no sabemos hasta qué punto son certeras, le sobrevinieron críticas como: "su argumento resulta suma y perfección de aberraciones, alambicado, casi grotesco pero sublime engendro. Obtiene carta de legitimación al ser motivo de discusión pública, por su temática morbosa y aspectos todavía no tratados en las radionovelas de su tiempo como el aborto, el cáncer y el racismo, y hasta conmueve al aquiescente clero de la época, pues uno de los personajes femeninos se acoge a la paz conventual" (9)

Algo sí es cierto, después de su salida al aire nada pudo obstaculizarle el camino a la cumbre de la novela radial. "(...) De radionovela logra metamorfosearse en fotonovela, telenovela, cine, historieta y libro. Gana eco en el folclor oral de los pueblos latinoamericanos. Da nombre a incontables niños nacidos durante la transmisión de sus capítulos, Ilamados Isabel Cristina o Alberto, como sus protagonistas." (10) Es noticia en la revista Time, que basada en su triunfo, demostrado en el record de audiencia, comenta: «Este acaramelado melodrama está siendo transmitido por 7 emisoras colombianas, de lunes a sábado» (11) y luego realiza una comparación entre la soap opera del principio y las derivaciones a que llega el género en América Latina, en las que Caignet tiene mucho que ver:

Caignet supo darle un nuevo giro a la radionovela, de acuerdo con el gusto latino, y logró un éxito sin precedentes. Supo retener el diario punto de tensión, el sollozo lastimero, una ausencia total de sutilezas y el sonoro e inquietante narrador. Trasladó la acción de la sala y la cocina, lugares en los que se desarrollaban las soap operas, a sitios tan exóticos como los cafetales de Palma Soriano. $\mathrm{Y}$ mientas las novelas en serie norteamericanas tienden a reunir los problemas sexuales, al menos en sus 
manifestaciones más vigorosas, Caignet se lanzó de lleno al campo de la pasión, al aborto y la ilegitimidad. (12)

Sobre estas dos creaciones diría Xiomara Fernández: "En la década del cuarenta aparecieron programas que hicieron historia, como la novela El derecho de nacer o la serie Chan Li Po. A la hora que salían al aire se paralizaban teatros y cines, y se encendía la radio para que la gente lo escuchara". (13).

El derecho de nacer abriría las puertas para la exportación de estos "novedosos" productos, como una carta de garantía de éxito. Veamos varios ejemplos que apoyan este planteamiento. "(...) la extensión de estos melodramas alcanza un punto culminante cuando se funda una empresa con capital cubano-mexicano, CUBA-MEX, para llevar al cine los argumentos radiales más exitosos. Uno de sus accionistas es el propio Caignet, con las ganancias por la transmisión azteca de "El derecho de nacer" como fondos iniciales. Desde 1951 «lo cubano es la onda» en los países del área, las radioemisoras adaptan sus trasmisores a la «técnica cubana». Tras la experiencia de nutrir sus programas con espectáculos de autores cubanos, las radioemisoras comprueban que la audiencia aumenta en forma inusitada. En muchos países los programas que alcanzan los mejores ratings provienen de Cuba: Tamakún, El Derecho de Nacer, El enemigo, Lo que pasa en el mundo, Los tres Villalobos, La Mentira, Yo no creo en los hombres, El dolor de ser pobres." (14)

¿Será el trasfondo económico lo único que se esconde detrás de la radionovela? ¿Qué mecanismo más grande hizo funcionar esta superestructura de "lágrimas"?

Cuando productos culturales como este se arraigan en el público, se empieza a pensar en ellos como una muestra de arte, más accesible al gusto popular y por ende más aceptado. Por eso los que deciden estudiar el fenómeno desde una perspectiva crítica, pueden percatarse que en realidad muy poco aporta. "Las primeras dramatizaciones radiales implantan un concepto cultural típico de la clase media asentada en las ciudades, pero dirigidas a sectores cada vez más amplios, en países eminentemente agrícolas. Se llega a la invención de una denominada «sensibilidad popular» que, casi siempre de modo natural, queda asumida como cultura urbana e impone una óptica citadina en países productores de materias primas y con poblaciones rurales mayoritarias." (15). Desde ese momento empieza un disgusto de la gente por sus raíces. La cultura que hasta ese momento había guiado sus vidas ya no les satisface más.

Las condiciones que brinda el medio encajaban perfectamente en las intenciones de los realizadores de convertir a las radionovelas en un producto comercial, y fueron muy bien 
aprovechados. "Ningún otro medio de comunicación colectiva tiene tantas posibilidades de utilizar el factor imaginal como la radio. (...) La capacidad y sentido de intimidad que produce la radio es uno de sus privilegios; la facilidad de transmisión y de recepción, su riqueza. El costo por millar de la radio es proporcionalmente más bajo al de cualquier otro medio masivo. (...) La radio es una fuente ligera y manejable de entretenimiento y de información instantánea. Por su flexibilidad es capaz de adaptarse a cambios de programación más rápido que cualquier otro medio y propicia la libertad de actividades diversas sin interrumpir a la gente, cosa que no es común en otros medios masivos (16)"

"El hábito magnifica tanto como la lejanía y hace ver eterno lo que es sólo temporal. A quienes están aficionados - ¿adictos?- a escuchar radionovelas, les será difícil pensar que no siempre las hubo (...)" (17). Acaparan la atención del público que hace suya la trama y sigue fielmente lo que en ellas aparece, aun en detrimento de su modo de actuar y de vivir. Las personas se identifican también con la posibilidad de lograr en sus vidas un desenlace feliz como en las novelas. En dependencia de cómo se mire sus efectos pueden ser negativos o positivos, pero no podemos negar que detrás hay una manipulación, la cual se hace evidente según Reinaldo González en que "(...) Dotados de insólitas buenas maneras y elegancia capaces de adquirirlas-, los protagonistas de origen humilde asumen las ideas y la disposición electiva de la clase superior, para acercárseles. Sus virtudes y sensibilidades los compulsan a cumplir objetivos cuya consumación incluye la necesidad de luchar...Luchan, sí, pero por demostrar que poseen las aptitudes individuales que se requieren para figurar en el escaño superior. Les proponen abandonar su propia clase y, por consiguiente, las razones que justificarían su lucha. Se les individualiza del conjunto. Se les descontextualiza. Hábitos ajenos, gratuidades, puros adornos de la personalidad-entendiendo como tal precisamente la despersonalización-, los alejan de conocimientos y enjuiciamientos de sus problemas verdaderos. Ahistoricismo y enajenación resultan cohonestados por la radionovela comercial y tradicional, al sobrevalorar el entretenimiento."

Un tanto relacionado con esto asombra encontrarse como «En ciertas aldeas peruanas pulen y pintan trozos de piedras imitando la apariencia de radios de transistores; los campesinos que son demasiado pobres para comprar una verdadera, llevan estas piedras consigo, en señal de status». (18)

No es difícil para un estudiante que se ha enfrentado al estudio de la Teoría Crítica, darse cuenta de que la radionovela es un instrumento de la industria cultural. Esta "forma parte de la estructura transnacional de poder, junto al control, interpretación y difusión de las noticias. Sus mecanismos de persuasión resultan sutiles y, en determinados sectores de nuestros países subeducados, más eficaces. Como otros vehículos del conjunto defienden el status quo, combaten los cambios estructurales, promueven una homogenización de los patrones 
de conducta y encausan las mentes hacia el consumismo. No se puede desatender la importancia de esos vehículos, pues se integran al sistema transnacional de comunicaciones, junto a las agencias de publicidad (...)" (19).

Pero, ¿qué elementos atraen a las personas hasta sus ondas como si fueran moscas? En un estudio realizado por Herta Herzog llamado "Motivaciones y satisfacciones de los que escuchan novelas" el autor comenta cuales podrían ser: "Parece que algunos oyentes gozan de las novelas simplemente como un medio de liberación emocional; gozan de las sorpresas felices o tristes. La oportunidad de expresar la agresividad también es una fuente de satisfacción. Por la carga de su propio problema, los oyentes afirman que les hace bien que otras personas también tienen congojas. Por una parte los oyentes gozan de los pesares de los personajes como compensación de sus propios problemas [...] Por otra parte, al identificarse ellos y sus problemas, que reconocen que son menos graves, con los angustiados héroes y heroínas de las historias, encuentran la oportunidad de agrandar sus propios padecimientos. [...] Una segunda y comúnmente reconocida forma de goce se refiere a las oportunidades que se tiene, al escuchar, de creer en lo que se desea [...] Una tercera y comúnmente insospechada forma de satisfacción se refiere a los consejos que se obtienen al escuchar las novelas radiales. Les gustan los relatos porque le explican las cosas al oyente que no sabe expresarse. Además les enseña formas adecuadas de comportamiento."

¿Quiénes fueron las principales víctimas de esta herramienta de la Industria cultural? Las mujeres, según Reinaldo González, vuelven a ser objeto de la tradicional manipulación a la que han sido sometidas desde mucho antes de surgir la novela radial. Esta, y los espacios que toman a la mujer como objeto central, responden a intereses estudiados. "Se dirigían fundamentalmente a un segmento de la población con una situación política social en desventaja respecto al resto de la sociedad. Exaltaban los problemas de las mujeres en un contexto donde ellas estaban absolutamente preteridas. Los anunciantes sabían que, a través de las novelas captaban a un determinado público, asiduo a un determinado horario.

Pero también hay que tener en cuenta que la mujer decide, tradicionalmente, por lo menos el $80 \%$ de las compras. Sobre todo las compras de los suministros del hogar no las decide el hombre, y mucho menos en la sociedad anterior. (...) ¿Quién compraba entonces el detergente o el jabón? A veces era incluso la lavandera de la casa. Toda la campaña de Candado iba destinada a las lavanderas, un ejército de mujeres que se ganaban la vida lavándoles la ropa a los que más tenían. (21).

La radionovela contribuyó a fijar estereotipos machistas en una región (Latinoamérica) que de por sí ya lo era. "La mujer se debate entre las categorías de indefensa, incomprendida, incógnita psicológica, expuesta a tentaciones...agredida por la bestialidad del hombre pero 
dependiente de él, porque «sólo el hombre puede comprenderla a fondo y salvarla en un mundo agresivo, cruel y despiadado»." (22).

Además de su tratamiento del sexo femenino, la radionovela presenta, como producto de la industria cultural, otros elementos criticables, sobre todo las maneras de abordar temas superfluos y de escasa variación, cuando cuestiones como la desnutrición, la mortalidad infantil con cifras alarmantes, las fuentes de ingreso cada vez más ineficientes, son de primer orden y deberían comunicarse por esta vía tan aceptada por el pueblo.

Su presencia dentro de la industria cultural no está aislada de otros productos de la misma: "argumentos radiofónicos pasan a la televisión, al cine, a los comics o historietas (muñequitos en Cuba), a la fotonovela, o llegan a la radio desde ellos.

(...) Dos grandes acontecimientos calzan esta afirmación: El derecho de nacer y simplemente María. Separados por varias décadas, ambos tienen la virtud de viajar de un país al otro del hemisferio y atravesar los mares para extender su «bondad» cultural. Ambos llegan a sus consumidores en casi todas las formas de la industria cultural moderna." (23)

El paso del tiempo trae innovaciones formales, pero no artísticas. Se amplían los límites de lo permisible -fundamentalmente en la utilización de un erotismo que roza lo pornográfico-, pero los significados se mantienen inflexibles. Aunque se incluyan variantes temáticas, la consideración que los empresarios tienen del auditorio es la misma: refractario a la novedad, a la innovación, a la provocación, al desorden, a la controversia, a la sutileza. Creen demostrado que el público prefiere caracteres de continuidad, con rasgos amables y divertidos. (24)

Para suerte de los actuales cubanos oyentes de las radionovelas la situación ha ido cambiando. Sus creadores "reconocen la sensibilidad popular a partir de estudios más sensatos y se proponen un mensaje novedoso en una forma amena. Al interesar a las mayorías, no las circunscriben al triángulo amoroso y no pretenden servirles la papilla de un producto deliberadamente concebido para ahorrarles esfuerzo intelectual. Esos aciertos tienen aceptación masiva porque establecen un diálogo inteligente con su auditorio. Pero todavía son escasos, en relación con la programación general que suele reducir las nuevas temáticas a los viejos esquemas formales, los recursos tradicionales del culebrón." (25)

Se decide contextualizar este análisis en Villa Clara, pues las fuentes primarias consultadas son estudios de audiencia realizados en esta provincia. Por tal motivo consideramos 
importante la opinión de Fernando González Castro, realizador de la emisora Provincial CMHW, uno de los villaclareños que han dedicado su vida a este género de la radiodifusión.

Fernando afirma que Cuba es uno de los pocos países del mundo que aún mantiene viva la Radio Novela, y que el formato del guión o libreto dramatizado ha sufrido su evolución como la Radio misma, sin embargo su esencia se ha mantenido y en la actualidad esa estructura ha devenido prácticamente uso exclusivo de los escritores cubanos que tributan a la programación dramatizada de Radio pues nuestro país fue el pionero de ésta y en la actualidad en el mundo es uno de los que mayor volumen de producción dramatizada realiza.

Al consultar el trabajo dedicado a "La audiencia de programas dramatizados en Villa Clara", se pudo constatar que el programa dramatizado preferido por un grupo de radioyentes encuestados resultó la novela; y quienes gustan de ella son preferentemente las mujeres. Este tipo de dramatizado quedó avalado por un $76 \%$ del total de los consultados que lo prefirieron a otros como humorísticos, policíacos, cuentos o aventuras.

En esta investigación sobresalen algunos de los resultados del sondeo de opinión de la programación de verano 2007 realizado por la emisora CMHW. Por los datos que esta ofrecía se pudo constatar que los menores de 30 no constituían un elemento significativo a la hora de estudiar los públicos que prefieren la radionovela. Se decidió entonces, realizar sesiones de trabajo, de aproximadamente 40 minutos de duración, con grupos de personas mayores de treinta años, estratificados por su ocupación laboral, con el objetivo de conocer los aspectos que mantienen viva la tradición de escuchar la radionovela, y los que atentan en su contra.

Hombres y mujeres hablaron por igual de sus gustos, condicionados muchas veces por otras responsabilidades. Así están los que ya no la oyen porque en el centro de trabajo no se lo permiten; porque influye negativamente en la concentración que deben tener para realizar las tareas profesionales; o porque aunque les gustaría, el tiempo no les alcanza pues deben emplearlo en los quehaceres hogareños. Otros, sencillamente, nunca tuvieron el hábito de escuchar la radio o interés por los productos mediáticos. Algunos que antes la escuchaban han dejado de hacerlo, porque otros medios han suplido el espacio dedicado a la radio y por tanto a la radionovela; o incluso se les ha roto el radio y perdieron el interés. De igual manera siempre está el que oye otros programas radiales en ese momento.

En el grupo de los que reconocieron la preferencia por este producto radial, los criterios aducen que se sientes atraídos por el reparto (los personajes), la actuación, el argumento (al cual catalogan de atractivo y bueno) y sus temas en algunas ocasiones divertidos y amenos. El entretenimiento que logra la radionovela es un elemento que muchos mencionaron, al igual que la capacidad que le atribuyen para atraer, brindar placer y relajar. En algunos el 
gusto llega como una influencia de grupos cercanos: compañeros de trabajo, vecinos, familia. Para otros es más fácil por la poca concentración que requiere el trabajo, bodegueros, amas de casa. Está también el que se considera aficionado o "viejo" en la acción de escucharlas y confiesa reconocerse como un novelero (a) de toda la vida.

En los grupos estudiados las mujeres de todo tipo de ocupación resaltaron como el público que prefiere más este género, con lo cual se corrobora la información sobre su preferencia expuesta anteriormente.

\section{Conclusiones}

Con desmanes e imperfecciones, cargada de detractores que en suma nunca superarán los que la defienden; la radionovela subsiste y augura permanecer por años en el panorama radiofónico cubano. La ausencia de recursos financieros, unida a la inexistencia en Cuba de canales televisivos con espacios para más dramatizados, nos conducen a creer en la subsistencia en la Mayor de las Antillas de la radionovela por encima de la telenovela. En el mundo comienzan a aparecer pequeños intentos por recuperar la producción de radionovelas, pero la megaindustria de la producción dramatizada en serie mantiene a raya tales intenciones. Mientras tanto nos queda velar porque además de proporcionar el placer de la enajenación, por esta vía transiten felices maneras de educar a la ciudadanía.

\section{Notas}

(1) González. R, 2002. pp. 87

(2) López. J, 2000. pp. 50

(3) González. R, 2002. pp. 64

(4) González. R, 2002. pp. 107

(5) O. L. López, 1998. pp. 34

(6) González. R, 2002. pp. 116

(7) González. R, 2002. pp. 131

(8) González. R, 2002. pp. 142

(9) González. R, 2002. pp. 158

(10) González. R, 2002. pp. 166

(11) González. R, 2002. pp. 170 
(12) González. R, 2002. pp. 186

(13) R. Garcés, 2005. pp. 57

(14) González. R, 2002. pp. 148

(15) González. R, 2002. pp. 122

(16) FIGUEROA, 1997: 389. pp. 39. (Extraído del artículo "De radio en radio: el escenario radiofónico", de los licenciados Dulce María García Dávila y José Gregorio Meza Tovar.)

(17) González. R, 2002. pp. 200

(18) González. R, 2002. pp. 214

(19) González. R, 2002. pp. 232

(20) González, R, 2002. pp. 181-215

(21) R. Garcés, 2005. pp. 45

(22) González. R, 2002. pp. 256

(23) González. R, 2002. pp.144

(24) González. R, 2002. pp. 260

(25) González. R, 2002. pp. 275

\section{Bibliografía}

Garcés, R. (2005). Los dueños del aire. Pablo de la Torriente Brau. pp.45-57

García, M. (2000). La audiencia de programas dramatizados en Villa Clara. Santa Clara: Departamento Metodológico y de Investigaciones Sociales. Radio provincial CMHW.

González, R. (2002). Llorar es un placer. La Habana: Letras Cubanas. pp.64-275

López, O. L. (1998). La radio en Cuba. Letras cubanas. pp.34-42

Rodríguez, E. N. (2001). Mi vida al desnudo. La Habana: Ediciones Unión.

Tovar, D. M. (s.f.). De radio en radio: el escenario radiofónico. Maracaibo.

López, J. I. (2000). Manual urgente para radialistas apasionados. La Habana: Pablo de la Torriente. pp.50

\section{Forma de citar este artículo en bibliografías}


TORRES FIGUEROA, O. Y GONZÁLEZ OCAÑA, E. (2011): "Radionovela: lágrimas, susurros y una felicidad siempre pospuesta"en Revista PANGEA, 2, páginas 159 a 170. Red Académica Iberoamericana de Comunicación. Recuperado el de de 2 de: www.revistapangea.org 